\title{
Irregular Firing and High-Conductance States in Spinal Motoneurons during Scratching and Swimming
}

\author{
Robertas Guzulaitis, ${ }^{1}$ Jorn Hounsgaard, ${ }^{1}$ and Aidas Alaburda ${ }^{2}$ \\ ${ }^{1}$ University of Copenhagen, Department of Neuroscience and Pharmacology, Panum Institute, Copenhagen DK 2200, Denmark, and ${ }^{2}$ Vilnius University, \\ Department of Neurobiology and Biophysics, Faculty of Natural Sciences, Vilnius LT 03101, Lithuania
}

Intense synaptic transmission during scratch network activity increases conductance and induces irregular firing in spinal motoneurons. It is not known whether this high-conductance state is a select feature for scratching or a property that goes with spinal motor network activity in general. Here we compare conductance and firing patterns in spinal motoneurons during network activity for scratching and swimming in an ex vivo carapace-spinal cord preparation from adult turtles (Trachemys scripta elegans). The pattern and relative engagement of motoneurons are distinctly different in scratching and swimming. Nevertheless, we found increased synaptic fluctuations in membrane potential, irregular firing, and increased conductance in spinal motoneurons during scratch and swim network activity. Our finding indicates that intense synaptic activation of motoneurons is a general feature of spinal motor network activity.

Key words: central pattern generators; high-conductance states; irregular firing; locomotion; motoneuron

Significance Statement

Neurons embedded in active neural networks can enter high-conductance states with irregular firing. This was previously shown for spinal motoneurons during scratching. Because scratching is highly specialized rhythmic behavior, it is not known whether high-conductance states and irregular firing are a peculiarity for motoneurons during scratching. Here, using intracellular recordings from motoneurons in an ex vivo carapace-spinal cord preparation from adult turtles, we demonstrate that irregular firing and high-conductance states are present not only during scratching but also during swimming. Our findings suggest that irregular firing and high-conductance states could be a general feature for motor behaviors.

\section{Introduction}

Central pattern generators (CPGs) are networks of neurons that generate rhythmic motor output in the absence of sensory feedback. To understand the origin of their rhythmicity, CPG networks with small number of neurons have been studied intensely (Grillner et al., 1998; Marder and Bucher, 2007). In these small-scale CPGs, intrinsic response properties of neurons play a key role for rhythmicity (Marder and Calabrese, 1996; Grillner, 2003). It was also thought that intrinsic properties of motoneurons would contribute to spike patterns in large-scale CPGs (Delgado-Lezama and Hounsgaard, 1999; Kiehn et al., 2000). This was challenged by the high-

Received Jan. 28, 2016; revised March 17, 2016; accepted April 19, 2016.

Author contributions: J.H. and A.A. designed research;R.G. and A.A. performed research; R.G. analyzed data; R.G. wrote the paper.

This work was supported by the Lundbeck Foundation and the Research Council of Lithuania Grant MIP-048/2013.

The authors declare no competing financial interests.

Correspondence should be addressed to Dr. Robertas Guzulaitis, University of Copenhagen, Department of Neuroscience and Pharmacology, Panum Institute, Blegdamsvej 3B Copenhagen DK 2200, Denmark. E-mail: r.guzulaitis@gmail.com.

DOI:10.1523/JNEUROSCI.0320-16.2016

Copyright $\odot 2016$ the authors $\quad 0270-6474 / 16 / 365799-09 \$ 15.00 / 0$ conductance states observed in motoneurons during scratching in an ex vivo carapace-spinal cord preparation from adult turtles (Alaburda et al., 2005; Berg et al., 2007). Highconductance states were observed earlier in the cortex (BorgGraham et al., 1998; Paré et al., 1998; Destexhe et al., 2003) and are thought to mediate irregular firing (Softky and Koch, 1993; Holt et al., 1996). Furthermore, spinal motoneurons fire irregularly during scratching (Berg et al., 2007, 2008) due to increased synaptic fluctuations in membrane potential and shunting intrinsic properties (Alaburda et al., 2005; Berg et al., 2007). However, high-conductance states and irregular firing in motoneurons were observed during scratching, which is a highly specialized motor behavior. The hind-limb scratch is a rhythmic motor behavior induced by somatosensory stimulation aimed at removing an irritant from the body surface (Stein, 2005). At least in cats, scratching and locomotion have been suggested to be generated by distinctly different network mechanisms (Frigon and Gossard, 2010). Therefore, network mechanisms derived from scratching cannot be directly applied to locomotion.

In the present study, we use a modified version of an ex vivo carapace-spinal cord preparation (Alaburda and Hounsgaard, 
2003) to record from motoneurons during scratch and swim network activity. We ask whether the high-conductance state is a peculiarity for motoneurons during scratching or a general feature for spinal CPGs in the turtle.

\section{Materials and Methods}

Ethical approval. The surgical procedures complied with Danish legislation and were approved by the controlling body under the Ministry of Justice.

An isolated ex vivo carapace-spinal cord preparation. Red-eared turtles (Trachemys scripta elegans) were obtained from Nasco. Surgical procedures were described previously (Alaburda and Hounsgaard, 2003). Briefly, turtles ( $n=11$, of either sex), $10-15 \mathrm{~cm}$ carapace length, were placed on crushed ice $2 \mathrm{~h}$ before surgery to induce drowsiness and reduce stress and pain by hypothermia. In this way, the head and neck could be protracted using minimal force. Brain functions were terminated immediately upon decapitation by crushing the head. The blood was substituted by perfusion through the heart with a Ringer's solution containing the following (in $\mathrm{mm}$ ): $120 \mathrm{NaCl}, 5$ $\mathrm{KCl}, 15 \mathrm{NaHCO}_{3}, 2 \mathrm{MgCl}_{2}, 3 \mathrm{CaCl}_{2}$, and 20 glucose, saturated with $98 \% \mathrm{O}_{2}$ and $2 \% \mathrm{CO}_{2}$ to obtain $\mathrm{pH}$ 7.6. To isolate the spinal cord, musculature and limbs were removed and transverse cuts were made rostral to spinal roots of D10 (or S2) and C6 spinal segments. The hip flexor (HF) motor nerve was exposed and cut.

Stimulation. A tactile stimulus on the body surface of a turtle elicits a rhythmic motor response termed scratching (Stein, 2005). During a scratch, the limb reaches toward and rubs against the stimulated site. In the ex vivo carapace-spinal cord preparation, scratch network activity was initiated by mechanically stimulating turtle carapace (M8-M9.5 dermatomes) with fire-polished glass rod mounted to the membrane of a loudspeaker controlled with a function generator (see Fig. 1, loudspeaker symbol). Stimulation of these dermatomes of the turtle carapace induces pocket scratching (Mortin et al., 1985), and for simplicity we will refer to it as scratching.

In vivo studies showed that electrical stimulation of descending contralateral dorsalateral funiculus (cDLF) of the turtle spinal cord elicits rhythmic limb movements similar to those observed during swimming (Lennard and Stein, 1977). Electrical stimuli (0.2-0.5 ms pulses of 0.5-2 $\mathrm{mA}$ intensity applied at $5-15 \mathrm{~Hz}$ frequency) for induction of swim network activity were applied to CDLF at C6 spinal segment using a bipolar concentric electrode (model TM33CCNON, World Precision Instruments) (see Fig. 1, yellow pipette).

Recordings. Intracellular recordings of neurons in current-clamp mode were performed with a Multiclamp 700B or Axoclamp-2A amplifier (Molecular Devices). Sharp glass electrodes from thin-walled borosilicate glass were filled with a mixture containing $0.9 \mathrm{M} \mathrm{CH}_{3} \mathrm{CO}_{2} \mathrm{~K}$ and $0.1 \mathrm{M} \mathrm{KCl}$. $\mathrm{KCl}$ was used to stabilize the $\mathrm{AgCl} / \mathrm{Ag}$ electrode potential and reduce drift. The range of electrodes resistance was 35-50 $\mathrm{M} \Omega$. Recording electrodes (see Fig. 1, gray pipettes) were positioned in the ventral horn, and intracellular recordings from neurons were performed in bridge mode. Motoneurons were identified by their location in the lateral part of ventral horn (electrode were positioned under visual guidance), size (low $\mathrm{R}_{\mathrm{M}},<70 \mathrm{M} \Omega$ ), height of APs $(>80 \mathrm{mV})$, stable membrane potential more negative than $-50 \mathrm{mV}$, and activity relation to nerve output. Data were sampled at $10 \mathrm{kHz}$ with analog-to-digital converter (Digidata 1440 or Digidata 1322A, Molecular Devices), displayed by means of Axoscope and Clampex software (Molecular Devices), and stored on a hard disk for later analysis.

The electroneurogram (ENG) of the HF nerve was recorded with a differential amplifier Iso-DAM8 (World Precision Instruments) using a suction electrode (see Fig. 1, blue pipette). The bandwidth was $100 \mathrm{~Hz}$ to $1 \mathrm{kHz}$.

Data analysis. Swim and scratch motor patterns were quantified by calculating duty cycle, mean intensity, and peak of HF nerve activity. First, the activity of the HF nerve was rectified and low-pass filtered $(3 \mathrm{~Hz}$ Gaussian) (see Fig. $2 A$, yellow trace, $B$, blue trace). HF nerve activity was divided into $\mathrm{HF}$ active (HF ON) and quiescence (HF OFF) phases by using $150 \%$ of baseline as threshold. The duty cycle was calculated as a

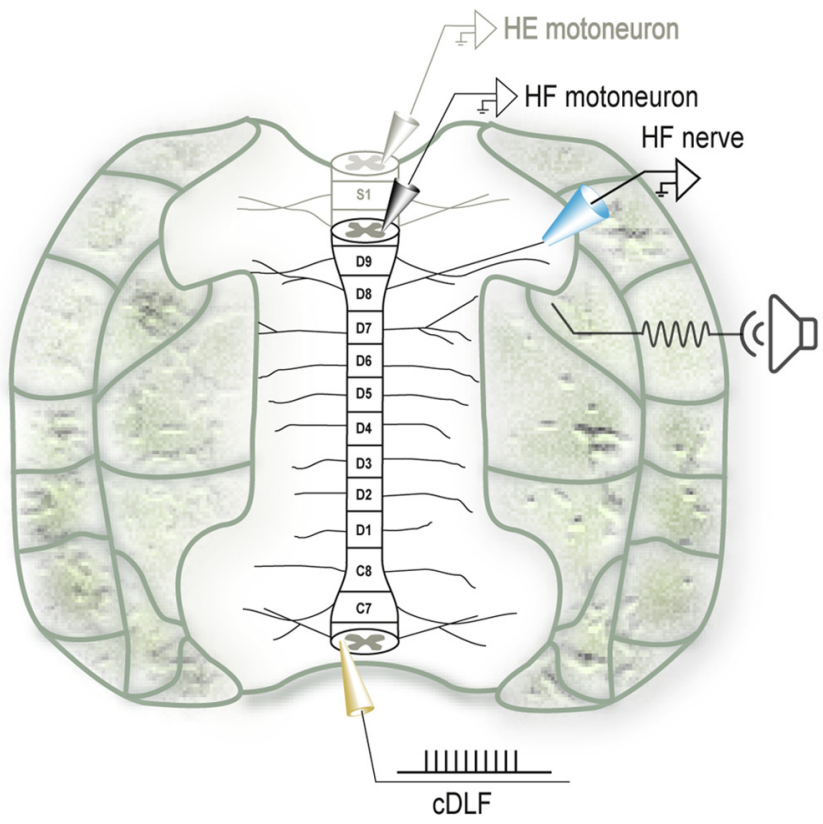

Figure 1. The ex vivo carapace-spinal cord preparation from the adult turtle. Schematics of the carapace and spinal cord (C6 to D10 or S2 segments). Scratch network activity was induced by mechanical stimulation of carapace (loudspeaker in right). Swim network activity was induced by electrical stimulation of CDLF at (6 segment (yellow pipette at bottom). Network activity was monitored by ENG recording from the HF motor nerve (blue pipette at right). Intracellular recordings from HF and HE motoneurons were performed at D10 or S2 spinal segments (gray pipettes in the top).

ratio between the HF ON phase and total cycle duration. Mean intensity for the HF nerve was calculated for HF ON periods as the area under the filtered HF trace divided by the duration of HF ON. Mean and peak intensities for the HF nerve were normalized.

Firing of neurons was evaluated by calculating interspike interval (ISI) between two adjacent action potentials (APs). Only ISIs within motor bursts were included in the analysis. The ratio of adaptation/ acceleration was calculated as a ratio between adapting $\left(\operatorname{ISI}_{i+1}>\right.$ ISI $\left._{i}\right)$ and accelerating $\left(\operatorname{ISI}_{i+1}<\right.$ ISI $\left._{i}\right)$ ISI pairs. Scatter in the $\operatorname{ISI}_{i} /$ ISI $_{i+1}$ plot was quantified by the Pearson correlation coefficient (Origin software; Microcal Software).

The irregularity of spiking was evaluated using coefficient of variation also known as CV2 (Holt et al., 1996) (Eq. 1) as follows:

$$
C V 2=\frac{2\left|I S I_{i}-I S I_{i+1}\right|}{I S I_{i}+I S I_{i+1}}
$$

CV2 was calculated for each AP and averaged across motor behavior or several responses to subthreshold current pulses. CV2 is presented as mean \pm SE (see Fig. 4Diii).

Fluctuations of the membrane potential $\left(V_{\mathrm{m}}\right)$ were evaluated as SD of $V_{\mathrm{m}}$ preceding APs. SD of $V_{\mathrm{m}}$ was calculated for a $10 \mathrm{~ms}$ time window (25 to $15 \mathrm{~ms}$ before APs).

Conductance of motoneurons was calculated by Ohm's low from $V_{\mathrm{m}}$ deflections induced by hyperpolarizing $100 \mathrm{~ms}$ current pulses applied at $5 \mathrm{~Hz}$ frequency (or $250 \mathrm{~ms}$ current pulses at $2 \mathrm{~Hz}$ ).

Data were analyzed statistically using Wilcoxon Signed Ranks Test for paired samples and Mann-Whitney Test for independent samples (Origin software; Microcal Software). Significance was accepted at $p<0.05$. If not stated otherwise, results are presented as mean \pm SD.

\section{Results}

\section{Scratching and swimming}

In this study, we used an ex vivo carapace-spinal cord preparation (Fig. 1) to study scratch and swim network activity. Rhythmic activity recorded in the HF motor nerve was evoked either by 
A

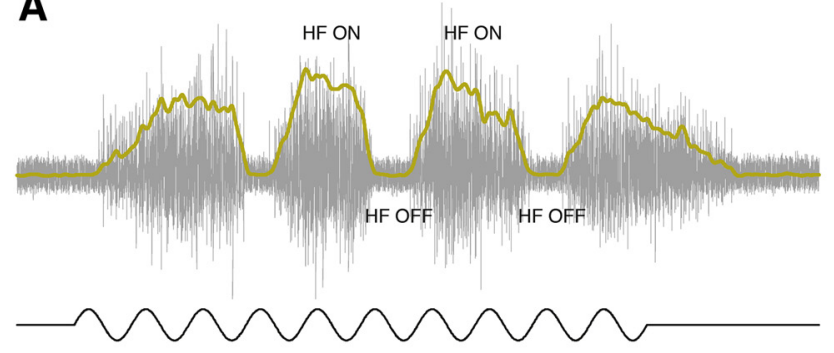

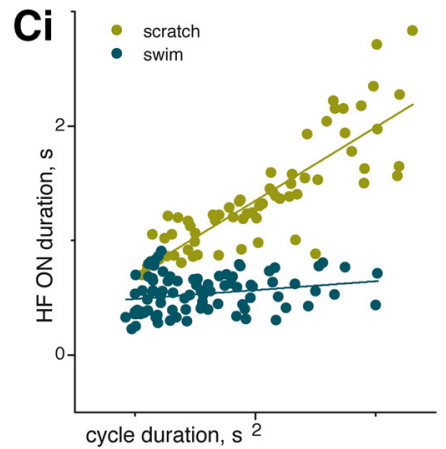

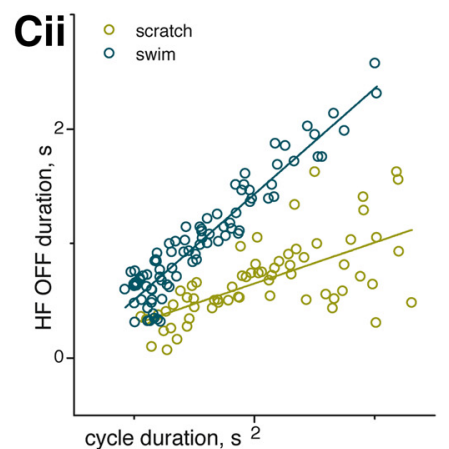

cycle duration, $\mathrm{s}^{2}$
B

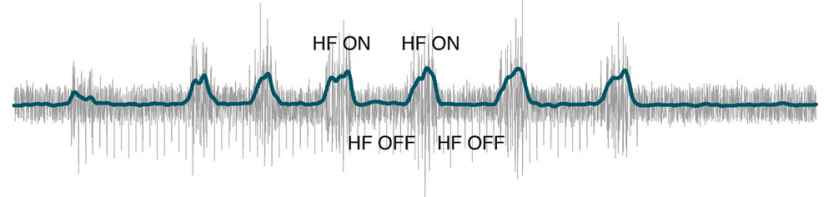

5s
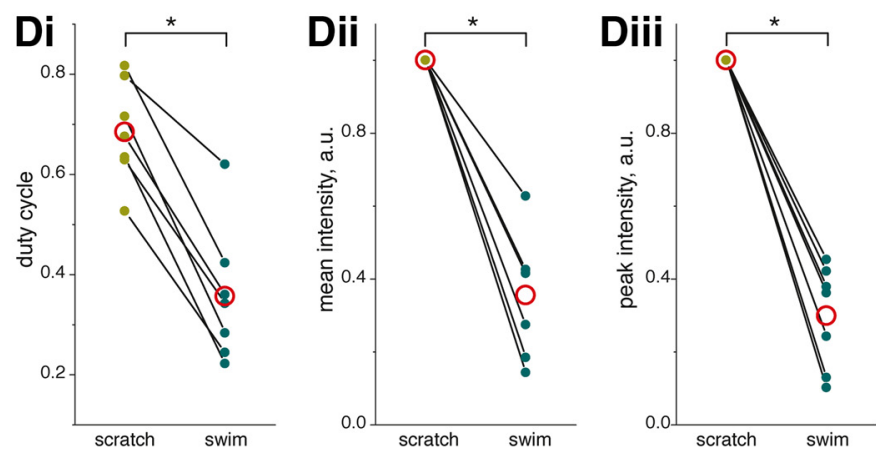

Figure 2. Swim and scratch motor patterns. Mechanical stimulation of the carapace ( $\boldsymbol{A}$, bottom) evokes fictive scratching observed as rhythmic activity in $\mathrm{HF}$ nerve ( $\boldsymbol{A}$, top). Electrical stimulation of CDLF ( $\boldsymbol{B}$, bottom) evokes fictive swimming ( $\boldsymbol{B}$, top). For quantification, the ENG signal was rectified and low-pass filtered (yellow for scratching in $\boldsymbol{A}$ and blue for swimming in $\boldsymbol{B}$ ). $\mathrm{HF}$ ON and $0 \mathrm{FF}$ phases were determined from filtered HF nerve activity. Relation between HF ON duration and total cycle duration in Ciand relation between HF OFF duration and total cycle duration in Cii. Duty cycle of HF activity is higher during scratching than during swimming (Di). In addition, mean (Dii) and peak (Diii) intensities of HF nerve activity are higher during scratching than during swimming. Di-Diii, Red circles represent mean. $A, B$, Top, Raw HF nerve activity in gray traces and rectified/filtered ENG signal in yellow for scratching and in blue for swimming; stimulus. ${ }^{*} p<0.05$.

mechanical stimulation of the carapace (Fig. $2 A$ ) or electrical stimulation of cDLF (Fig. 2B).

To further analyze the motor patterns of fictive swimming and scratching, the activity of HF nerve was rectified and filtered (Fig. $2 A$, yellow trace, $B$, blue trace). We noticed that cycle duration varied within individual motor responses and between responses. The cycle duration is regulated by changes in either the flexor or the extensor phase duration dependent on the type of behavior (Frigon and Gossard, 2010). During scratching, the duration of the HF ON phase covaried with cycle duration $(r=0.84, p<$ 0.001 , Pearson correlation coefficient, slope $=0.64, n=65$ motor cycles) but not during swimming $(r=0.24, p=0.025$, Pearson correlation coefficient, slope $=0.08, n=89$ motor cycles) (Fig. 2Ci). The same relation was tested for the HF OFF phase. HF OFF duration covaried with cycle duration during both scratching $(r=0.65, p<0.001$, Pearson correlation coefficient, slope $=$ $0.36, n=65$ motor cycles) and swimming $(r=0.95, p<0.001$, Pearson correlation coefficient, slope $=0.92, n=89$ motor cycles) (Fig. 2Cii). This demonstrates that cycle duration during scratching is determined by both HF ON and HF OFF durations. In contrast, the duration of HF OFF but not HF ON determines the cycle duration during swimming.

Next, the duration and intensity of HF nerve activity during swimming and scratching were compared. The duration of the $\mathrm{HF}$ cycle was evaluated as duty cycle (i.e., ratio between HF ON and total cycle duration). Duty cycle of HF nerve activity during scratching ( $0.69 \pm 0.1, n=7$ ex vivo preparations) is $\sim 2$ times higher than during swimming $(0.36 \pm 0.14, p=0.016$, Wilcoxon Signed Ranks Test, $n=7$ ex vivo preparations) (Fig. $2 D i$ ). HF intensity was quantified as mean and peak intensities (see Materials and Methods). Both mean ( $0.36 \pm 0.17, p=0.016$, Wilcoxon Signed Ranks Test, $n=7$ ex vivo preparations) and peak
$(0.3 \pm 0.14, p=0.016$, Wilcoxon Signed Ranks Test, $n=7$ ex vivo preparations) HF intensities are lower during swimming than during scratching (Fig. 2Dii,Diii).

To summarize, mechanical stimulation of the carapace and electrical stimulations of cDLF evoke distinct rhythmic motor behaviors as reflected in HF motor nerve activity. Even though both behaviors are rhythmic, HF activity is much shorter and of lower intensity during swimming than during scratching. Our results are in line with previous findings in vivo (Juranek and Currie, 2000) and probably reflect fundamentally different biomechanics during the two behaviors: the power stroke is produced in the HF phase during scratching and in the hip extensor (HE) phase during swimming (Stein, 2005).

\section{Motoneuron activity during scratching and swimming}

Motoneurons receive convergent synaptic input from the premotor network. Therefore, intracellular recordings from motoneurons provide information not only about intrinsic properties but also illuminate properties of the premotor network as reflected in the postsynaptic activity. Neurons recorded in the ex vivo carapacespinal cord preparation show rhythmic depolarizations of $V_{\mathrm{m}}$ during scratching and swimming (Fig. 3). Occasionally, when $V_{\mathrm{m}}$ reaches threshold, APs are generated. Neurons activated in phase with HF nerve were called putative HF motoneurons (or just HF motoneurons) (Fig. 3Ai,Bi) and neurons activated out of phase with HF nerve were called putative HE motoneurons (or just HE motoneurons) (Fig. 3Aii,Bii). Twenty-one motoneurons were recorded in total: $6 \mathrm{HF}$ motoneurons in D10 preparations and $2 \mathrm{HF}$ and $13 \mathrm{HE}$ motoneurons in S2 preparations. The separation of motoneuron pools in different preparations is compatible with the anatomical demonstration that the majority of HF motoneurons are located in 
Ai

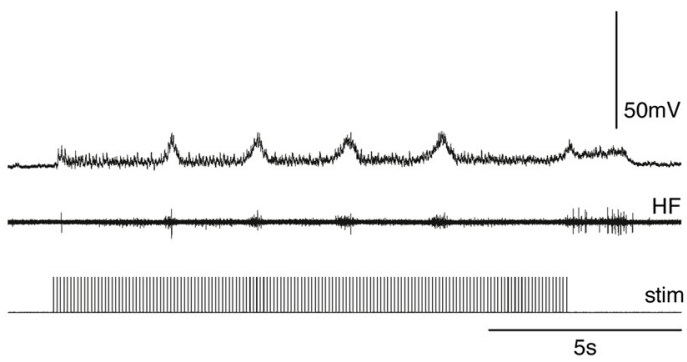

Aii

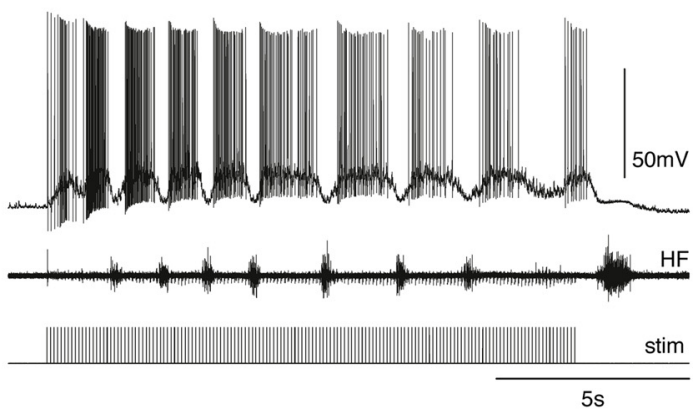

Bi

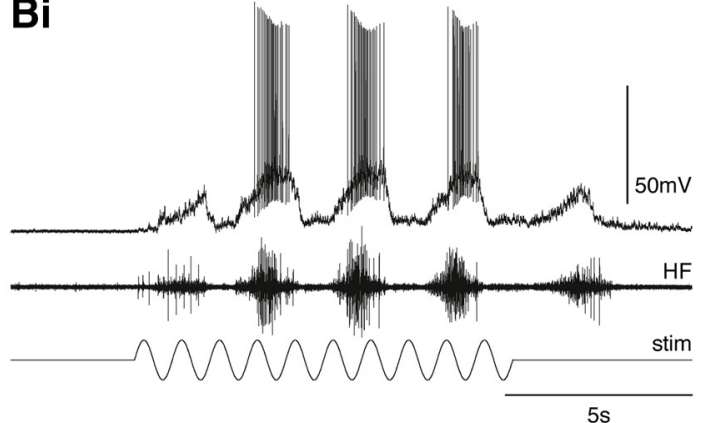

Bii

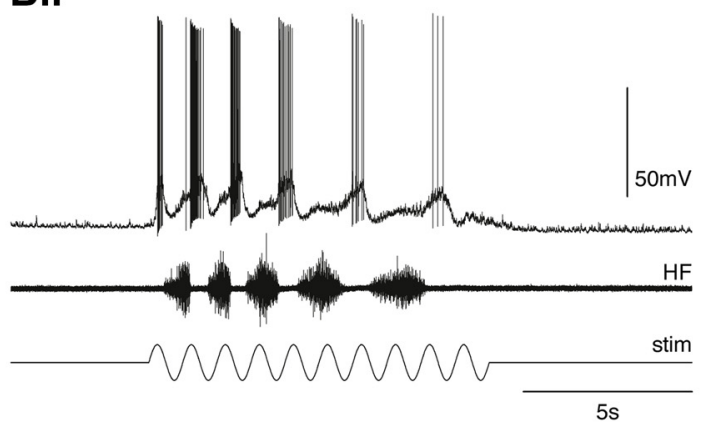

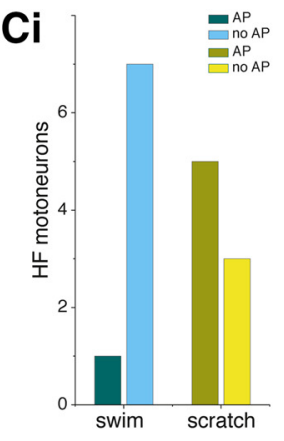

Cii

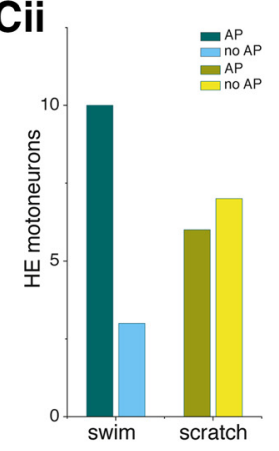

Figure 3. Activity of HF and HE motoneurons during swimming and scratching. Electrical stimulation of CDLF (Ai, Aii, bottom) evokes fictive swimming with characteristic short bursts in HF nerve ( $\boldsymbol{A i}$, Aii, middle). Example of HF motoneuron shows subthreshold depolarization of $V_{\mathrm{m}}$ in phase with HF nerve activity (Ai). In contrast, HE motoneuron shows depolarization of $V_{\mathrm{m}}$ with intense spiking out of phase with HF nerve activity (Aii). Mechanical carapace stimulation (Bi, Bii, bottom) evokes fictive scratching with relatively long HF nerve bursts (Bi, Bii, middle). Both HF (Bi) and $\mathrm{HE}$ (Bii) motoneurons are depolarized and fire APs during scratching. The majority of HF motoneurons show only subthreshold depolarization without APs during swimming, whereas more than half of those HF motoneurons generate APs during scratching (Ci). In contrast, most of HE motoneurons show suprathreshold activation with APs during swimming, whereas only approximately half of those HE motoneurons generate APs during scratching (Cii). Dark bars represent motoneurons generating APs during motor output. Light bars represent motoneurons with no APs during motor output. Ai, Bi, Recordings are from the same HF motoneuron. Aii, Bii, Recordings are from the same HE motoneuron. Ai, Aii, Bi, Bii, From the top, $V_{m}$ of motoneuron; HF nerve activity; stimulus.

D8-D10 segments, whereas HE motoneurons are distributed more caudally in sacral segments (Ruigrok and Crowe, 1984).

In agreement with HF motor nerve activity, we observed that the majority of HF motoneurons (7 of 8) were not active (did not generate APs) during swimming, whereas 5 of them were active (generated APs) during scratching (Fig. $3 \mathrm{Ai}, \mathrm{Bi}, \mathrm{Ci}$ ). In contrast, most of HE motoneurons (10 of 13) were active during swimming and approximately half ( 6 of 13 ) of them were active during scratching (Fig. 3Aii,Bii,Cii).

\section{Firing patterns of motoneurons during scratching and swimming}

In turtle motoneurons, the regular firing pattern evoked by a constant depolarizing current applied through the recording electrode at rest is replaced by irregular firing during scratch network activity (Berg et al., 2007, 2008). Here we compare the regularity of firing evoked during scratching, swimming, and injection of a depolarizing current pulse at rest.

The motoneuron shown in Figure $4 A i, B i$ generated several APs in each motor cycle during swimming and scratching. Variable ISIs were observed during swimming (Fig. 4Aii) and scratching (Fig. 4Bii). In addition, we tested firing patterns of motoneurons in response to suprathreshold current pulses (Fig. $4 \mathrm{Ci}, \mathrm{Cii}$ ). The intensity of current pulses was selected to evoke APs with mean firing rates $(39.9 \pm 5.54 \mathrm{~Hz}, n=6$ motoneurons $)$ similar to mean firing rates observed during swimming (48.93 \pm $22.63 \mathrm{~Hz}, n=9$ motoneurons $)$ and scratching $(45.71 \pm 15.76 \mathrm{~Hz}$, $n=9$ motoneurons).

First, the relation between the duration of successive ISIs was plotted (Fig. 4Aiii,Biii,Ciii), and the ratio between acceleration $\left(\right.$ ISI $_{i+1}<$ ISI $\left._{\mathrm{i}}\right)$ and adaptation $\left(\right.$ ISI $_{i+1}>$ ISI $\left._{\mathrm{i}}\right)$ was calculated. Motoneurons do not show strong adaptation or acceleration during either swimming (ratio $=0.91 \pm 0.13, n=9$ motoneurons) or scratching (ratio $=0.97 \pm 0.21, n=9$ motoneurons), and no differences were observed between these two behaviors (Fig. 4Di) ( $p=0.69$, Mann-Whitney Test). However, motoneurons show strong adaptation when current pulses are applied (ratio $=0.35 \pm 0.15, n=6$ motoneurons) compared with swimming $(p<0.001$, Mann-Whitney Test) and scratching $(p<$ 0.001, Mann-Whitney Test) (Fig. 4Di).

The regularity of spiking was evaluated as correlation between successive ISIs (Fig. 4Dii). Correlation during current pulse injection (0.65 $\pm 0.16, n=6$ motoneurons) was significantly higher than during swimming $(0.16 \pm 0.2, n=9$ motoneurons, $p<0.001$, Mann-Whitney Test) or scratching $(0.4 \pm 0.16, n=9$ motoneurons, $p=0.012$, Mann-Whitney Test). In addition, the correlation during swimming was lower than during scratching ( $p=0.01$, Mann-Whitney Test) indicating higher irregularity of firing during swimming.

Because motoneurons possess spike frequency adaptation, classical coefficient of variation as a measure of irregularity is not applicable. Instead, we used CV2 (Holt et al., 1996) as a measure of irregularity. CV2 is less sensitive to slow changes of firing rates. CV2 during firing evoked by depolarizing current pulses $(0.25 \pm$ $0.03, n=6$ motoneurons) was significantly lower than during swimming $(0.48 \pm 0.09, n=9$ motoneurons, $p<0.001$, MannWhitney Test) and scratching $(0.41 \pm 0.1, n=9$ motoneurons, $p<0.001$, Mann-Whitney Test; Fig. 4Diii). There was no difference between CV2 during swimming and scratching $(p=0.16$, Mann-Whitney Test). 
Ai

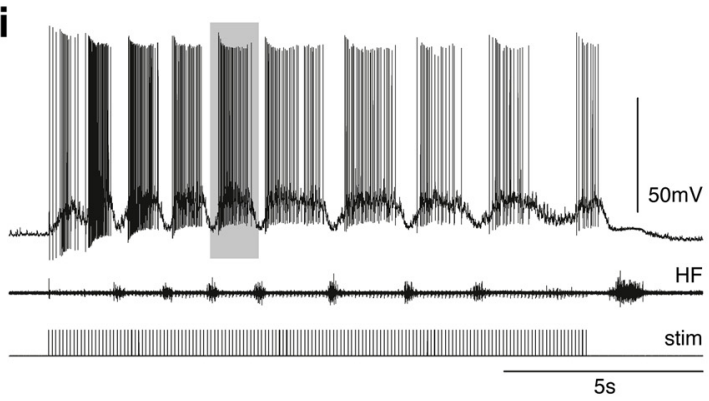

$B i$

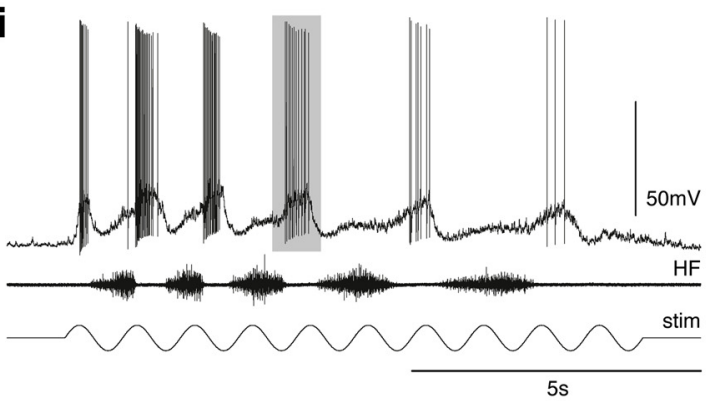

Aii
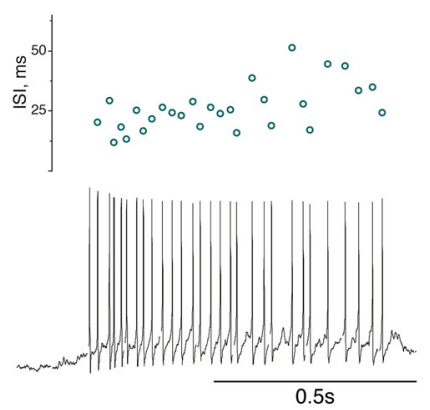

Bii

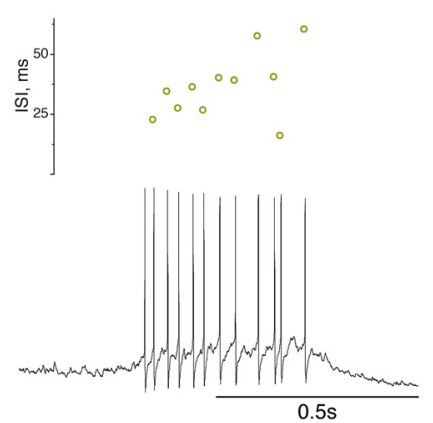

Cii

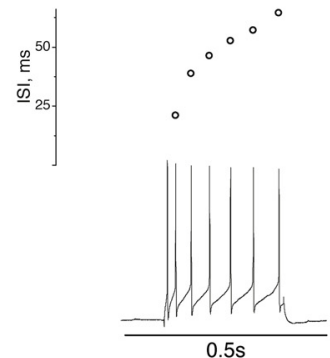

Aiii

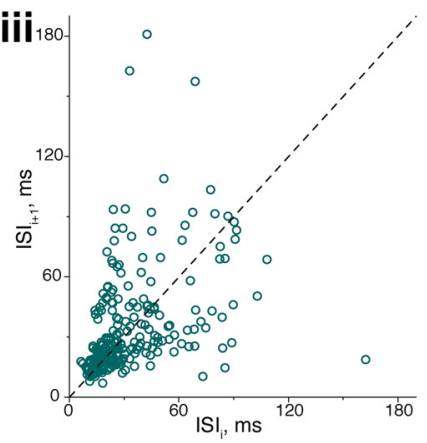

Biii

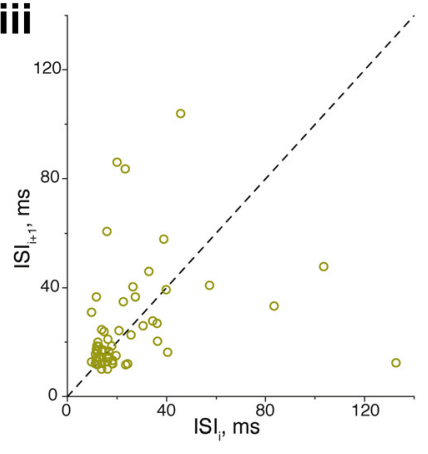

Ci

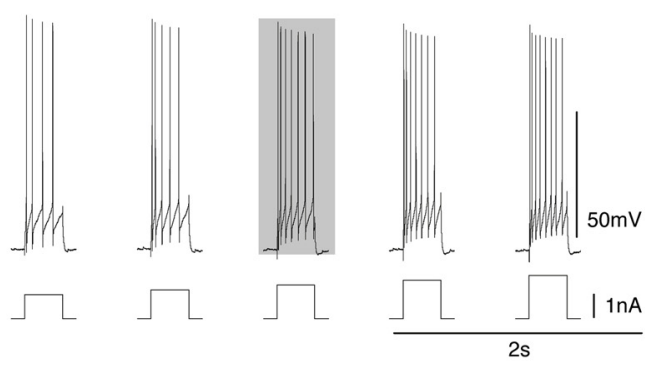

Dii

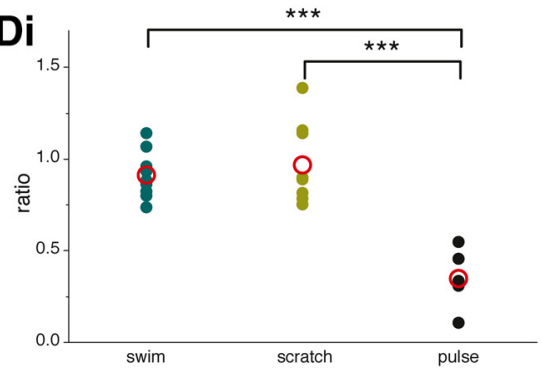

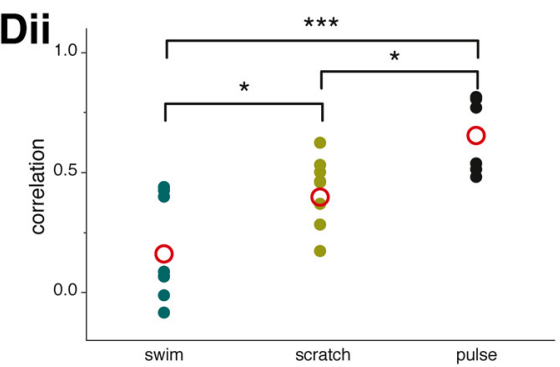

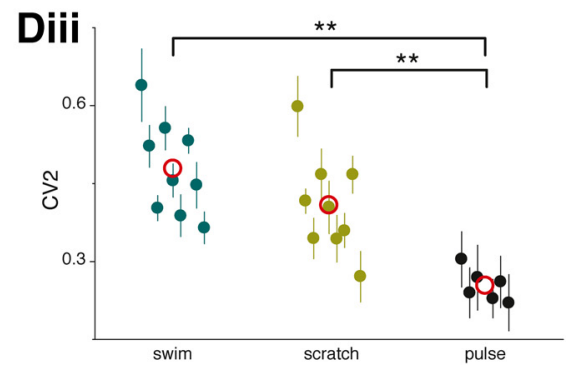

Figure 4. Firing of motoneurons during swimming, scratching, and depolarizing current pulse. Firing patterns of motoneurons during swimming (Ai), scratching (Bi), and depolarizing current pulses ( $\boldsymbol{C}$ i) were tested. ISI was calculated for every pair of APs. Ai, Bi, Ci, Episodes with APs in gray boxes are expanded in Aii, Bii, and Cii, and ISI for every AP is shown at the top. Relation between the successive ISIs (Aiii, Biii, Ciii) was used for calculating the ratio between adaptation $\left(\left.I S\right|_{i}<|S|_{i+1}\right.$ ) and acceleration $\left(\left.I S\right|_{i+1}<|S|_{i}\right)$ (Di) and correlation coefficient (Dii). Regularity of firing was also measured as coefficient of variation (CV2) during scratching, swimming, and depolarizing current pulse (Diii) and plotted as mean \pm SE. Di, Dii, Diii, Red circles represent mean. Ai, Bi, Ci, From the top, $V_{\mathrm{m}}$ of motoneuron; HF nerve activity; stimulus. Aii Bii, Cii, From the top, ISI of AP and $V_{\mathrm{m}}$ of motoneuron. ${ }^{*} p<0.05$; ${ }^{* *} p<0.01 ;{ }^{* * *} p<0.001$.

To summarize, our findings demonstrate that intrinsic spiking of motoneurons is much more regular than during swimming and scratching. Our findings confirm irregular firing during scratch network activity (Berg et al., 2007, 2008) and demonstrate similarly irregular firing during swim network activity.

\section{$V_{\mathrm{m}}$ fluctuations during scratching and swimming}

The level of $V_{\mathrm{m}}$ fluctuations increases in turtle motoneurons during scratching (Alaburda et al., 2005; Petersen et al., 2014). Here, $V_{\mathrm{m}}$ fluctuations during swimming (Fig. 5A), scratching (Fig. 5B), and current pulse injection (Fig. $5 C$ ) were compared. Fluctua- tions of $V_{\mathrm{m}}$ were evaluated when the motoneuron generates APs. SD of $V_{\mathrm{m}}$ was calculated just before APs are generated (Fig. 5, gray squares). SD of $V_{\mathrm{m}}$ during current pulse injection (1.59 \pm 0.42 $\mathrm{mV}, n=5$ ) was significantly smaller than during swimming $(2.97 \pm 0.56 \mathrm{mV}, n=9$ motoneurons, $p=0.003$, MannWhitney Test) and scratching (3.59 $\pm 0.69 \mathrm{mV}, n=9$ motoneurons, $p=0.003$, Mann-Whitney Test) (Fig. $5 D$ ). No significant difference ( $p=0.08$, Mann-Whitney Test) was found between $\mathrm{SD}$ of $V_{\mathrm{m}}$ during scratching and swimming.

Our findings show that the synaptic input from the premotor network gives rise to a very similar increase in $V_{\mathrm{m}}$ fluctu- 
ations in individual motoneurons during swim and scratch network activity.

\section{Conductance of motoneurons during scratching and swimming}

Conductance increases substantially in cortical neurons during network activity (Paré et al., 1998; Destexhe et al., 2003; Monier et al., 2008). A similar increase in conductance was demonstrated in turtle motoneurons during scratch network activity (Robertson and Stein, 1988; Alaburda et al., 2005; Berg et al., 2008). To determine whether conductance increase in motoneurons extends to other spinal network activities, we compared conductance in the same motoneurons during scratch and swim network activity.

The conductance was estimated by injecting hyperpolarizing current pulses into motoneurons before and during network activity (Fig. 6Ai,Aii). To quantify conductance change $V_{\mathrm{m}}$ before and during hyperpolarizing current injection were superimposed (Fig. 6Bi,Bii, light color traces) and averaged (Fig. 6Bi,Bii, darker color traces). The same current pulse induces higher $V_{\mathrm{m}}$ deflection at rest (Fig. 6Bi,Bii, left) than during swimming (Fig. 6Bi, right) and scratching (Fig. 6Bii, right), indicating conductance increase during network activity. Conductance during swimming (84.36 $\pm 29.69 \mathrm{nS}$, $n=21$ motoneurons) increases compared with resting conductance $(52.06 \pm 19.26$ $\mathrm{nS}, n=21$ motoneurons, $p<0.001$, Wilcoxon Signed Ranks Test) (Fig. 6Ci). In addition, conductance during scratching (83.01 $\pm 28.35 \mathrm{nS}, n=21$ motoneurons) is also higher than conductance at rest (50.85 $\pm 18.36 \mathrm{nS}, n=21$ motoneurons, $p<0.001$, Wilcoxon Signed Ranks Test) (Fig. 6Cii). This finding demonstrates that synaptic activity during swimming and scratching increases conductance of motoneurons by $\sim 60 \%$.

Conductance during scratching and swimming is higher than conductance at rest in all tested motoneurons (all data points are above diagonal in Fig. 7A). In addition, there is no difference between the relative conductance increase during swimming (32.3 \pm $23.25 \mathrm{nS}, n=21$ motoneurons) and scratching (32.16 $\pm 18.43 \mathrm{nS}$, $n=21$ motoneurons, $p=0.63$, Wilcoxon Signed Ranks Test) (Fig. $7 B)$. Conductance increase was tested for $\mathrm{HF}$ and $\mathrm{HE}$ motoneurons separately (Fig. 7C). Conductance increase in HF motoneurons during swimming (26.38 $\pm 22.61 \mathrm{nS}, n=8$ motoneurons) was not different ( $p=0.21$, Mann-Whitney Test) from conductance increase in HE motoneurons during swimming (35.95 $\pm 23.77 \mathrm{nS}, n=$ 13 motoneurons). In addition, conductance increase in HF motoneurons during scratching $(32.56 \pm 23.67 \mathrm{nS}, n=8$ motoneurons) was not different ( $p=0.64$, Mann-Whitney Test) from conductance increase in $\mathrm{HE}$ motoneurons during scratching (31.91 $\pm 15.46 \mathrm{nS}, n=13$ motoneurons).

Next, we compared conductance increase in spiking vs nonspiking motoneurons (Fig. 7D). There is a tendency of
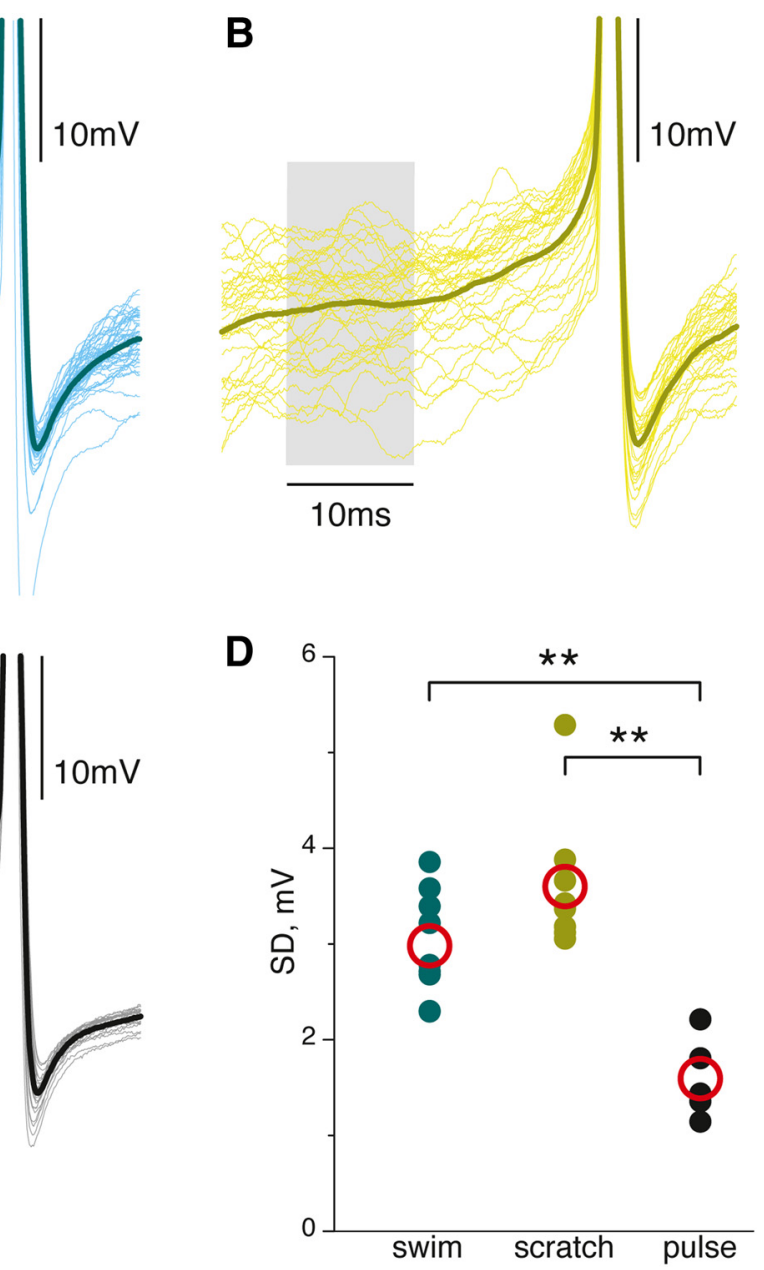

Figure 5. Fluctuations of $V_{m}$ during swimming, scratching, and depolarizing current pulse. Episodes of $V_{m}$ with APs during swimming $(\boldsymbol{A})$, scratching $(\boldsymbol{B})$, and current pulse injections $(\boldsymbol{C})$ were superimposed (light color traces), and mean was calculated (dark color traces). SD of $V_{m}$ before AP (25-15 ms before AP, gray area) was compared during swimming, sents raw superimposed $V_{m}$ traces, and dark color represents mean $V_{m}$ (blue for swimming, yellow for scratching, and gray for current pulse injections). ${ }^{* *} p<0.01$.

higher conductance increase in spiking motoneurons (36.9 \pm $22.23 \mathrm{nS}, n=22$ motoneurons for swimming or scratching) than in nonspiking motoneurons $(27.09 \pm 18.09 \mathrm{nS}, n=20$ motoneurons for swimming or scratching). However, the difference is not significant ( $p=0.11$, Mann-Whitney Test), demonstrating that even motoneurons not actively contributing to motor behavior receive intense synaptic input.

We noted that the conductance increase during scratching correlated with conductance increase during swimming $(r=$ $0.63, p=0.002$, Pearson correlation coefficient, $n=21 \mathrm{mo}-$ toneurons) (Fig. $7 E$ ). This demonstrates that conductance of motoneurons increases to the same degree during swimming and scratching, suggesting that synaptic intensity covary in two distinctly different motor network activities. One possibility could be that conductance increase during network activity scales with resting conductance. However, we did not find correlation between conductance at rest and relative conductance increase during swimming $(r=-0.03, p=0.88$, Pearson correlation coefficient, $n=21$ motoneuron) or scratching ( $r=0.19, p=0.42$, Pearson correlation coefficient, $n=21$ motoneuron) (Fig. $7 F$ ). This suggests that the increase 
Ai
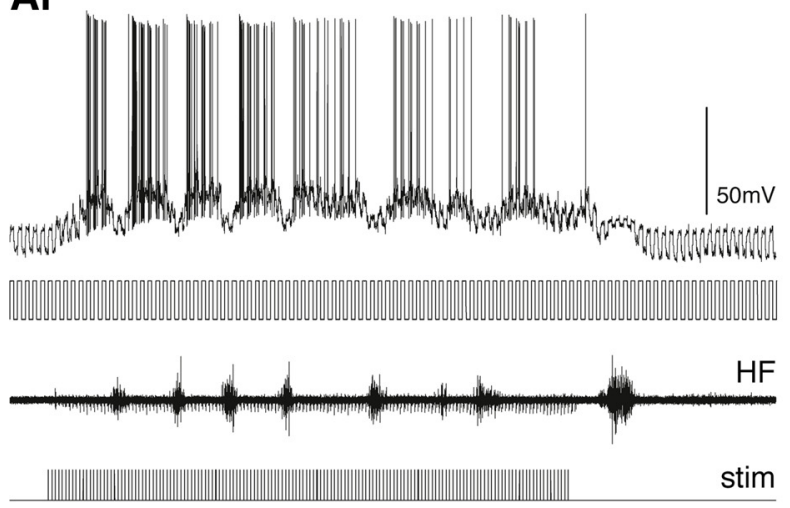

$5 s$

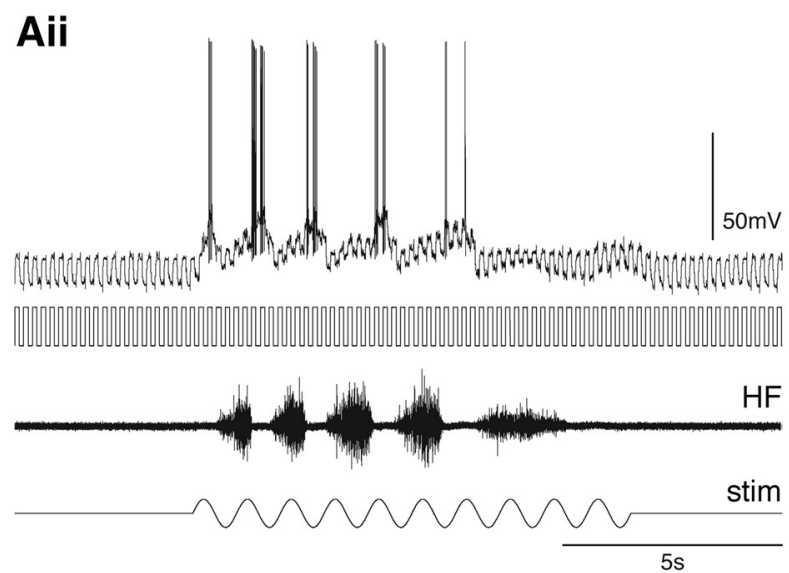

Bi

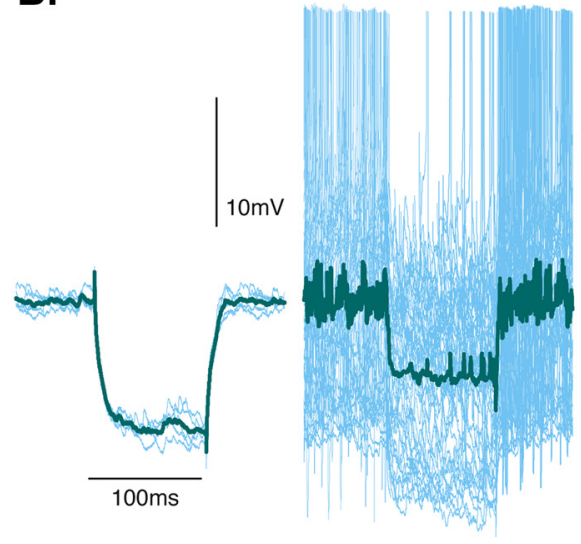

Bii

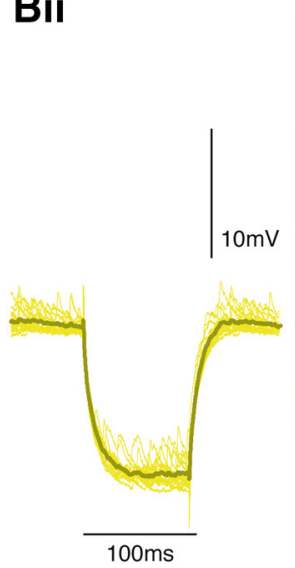

Ci

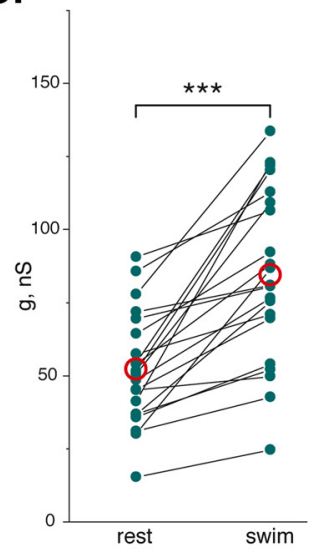

Figure 6. Conductance of motoneurons during swimming and scratching. Conductance during swimming (Ai) and scratching (Aii) was obtained from $V_{\mathrm{m}}$ response to hyperpolarizing current pulses $(-0.5 \mathrm{nA})$. Episodes of $V_{\mathrm{m}}$ before and during negative current pulse injection were superimposed (light blue and yellow traces) and averaged (dark blue and yellow traces). $V_{\mathrm{m}}$ deflection to injected current pulse is smaller during swimming (Bi, right) and scratching (Bii, right) than at rest (Bi, Bii, left). Conductance of motoneurons increases during swimming ( $\boldsymbol{C}$, red circles represent mean) and scratching (Cii, red circles represent mean). Bi, $\boldsymbol{B}$, Spikes are trimmed. $\mathbf{A i}, \mathbf{A i i}$, Top, $V_{\mathrm{m}}$ of motoneuron; current pulses injected; HF nerve activity; stimulus. ${ }^{* *} p<0.001$.

in conductance during spinal network activity is determined by other mechanisms rather than size of the motoneuron.

\section{Discussion}

Modification of the ex vivo turtle carapace-spinal cord preparation enabled us to study scratch and swim network activities. Although scratching and swimming are distinct motor behaviors, the underlying network activities share certain properties. We found that motoneurons receive intense synaptic activity during both scratching and swimming, which is reflected in a significant increase in $V_{\mathrm{m}}$ fluctuations and conductance. The conductance increase during scratching is highly correlated with the conductance increase during swimming in the same motoneurons. In addition, motoneurons activated during both scratching and swimming show irregular firing, which is a characteristic feature of intense synaptic activity.

Analysis of HF nerve recordings uncovered differences between scratch and swim network activities. HF activity is much longer and more intense during scratching than during swimming. In addition, cycle duration during scratching is regulated by parallel changes in HF ON and HF OFF duration, whereas cycle duration during swimming is mainly regulated by changes in HF OFF duration. These observations are in agreement with previous findings in vivo (Juranek and Currie, 2000).

In this study, it was demonstrated that turtle spinal motoneurons fire irregularly during scratch and swim network activities. It is known that turtle motoneurons generate regular spike trains with adaptation when stimulated with suprathreshold current injections (Hounsgaard et al., 1988), whereas high irregularity is observed during scratching (Berg et al., 2007, 2008). Irregular firing is a characteristic feature of cortical neurons recorded in vivo (Noda and Adey, 1970; Softky and Koch, 1993; Holt et al., 1996). Although endogenous mechanisms account for some degree of irregular firing (Azouz and Gray, 1999; Faisal et al., 2008), the major origin is thought to be uncorrelated concurrent inhibition and excitation (Softky and Koch, 1993; Shadlen and Newsome, 1998; Salinas and Sejnowski, 2000). Irregular firing was also observed in human motoneurons (Gandevia et al., 1990; Macefield et al., 1993; Vaillancourt et al., 2003) and shown to produce higher contractile responses in innervated muscles (Leitch and Macefield, 2014).

Synaptic input is reflected in $V_{\mathrm{m}}$ fluctuations. Therefore, variation in $V_{\mathrm{m}}$ may serve as an indicator of synaptic intensity (Paré et al., 1998; Guzulaitis et al., 2013; Petersen et al., 2014). Here we found that $V_{\mathrm{m}}$ of motoneurons is more variable during swimming and scratching than at rest. The variation in the $V_{\mathrm{m}}$ of motoneurons during scratching found in this study is comparable with previous observations (Petersen et al., 2014) and is not different from $V_{\mathrm{m}}$ fluctuations during swimming.

Irregular firing and fluctuations of $V_{\mathrm{m}}$ is often associated with high-conductance states (Paré et al., 1998; Destexhe et al., 2003). 
A
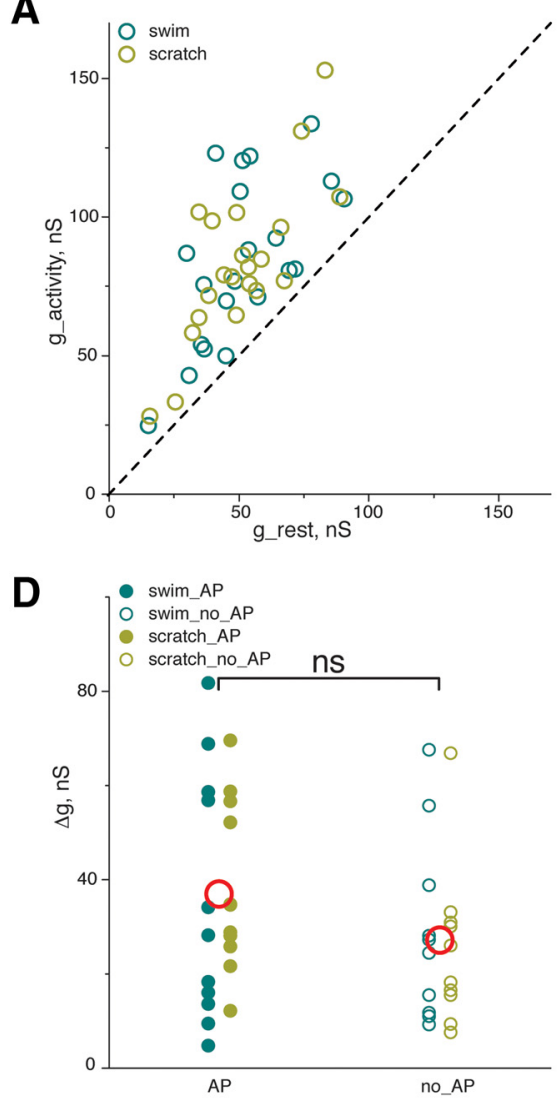

B
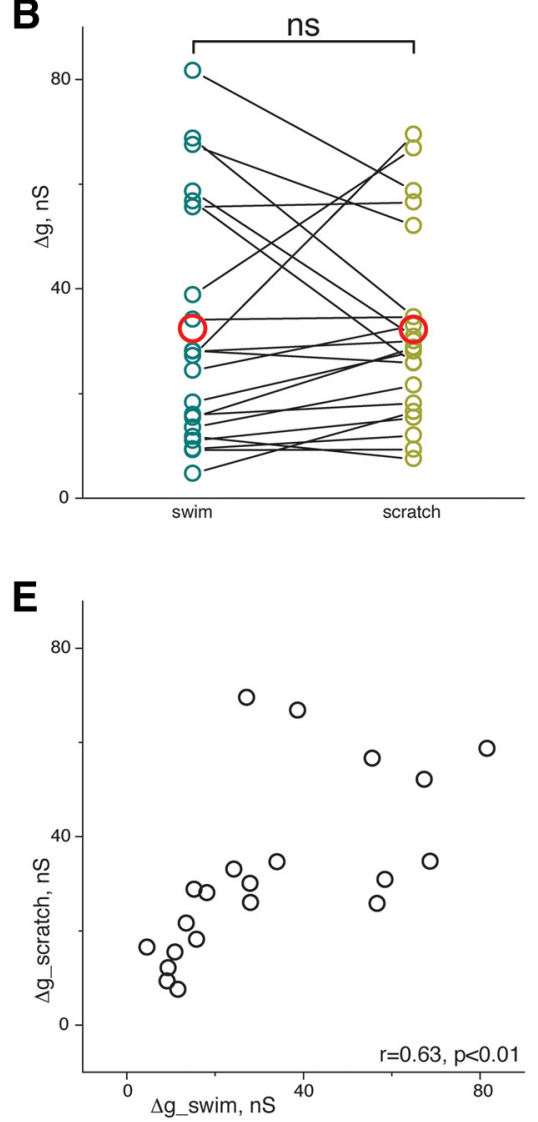

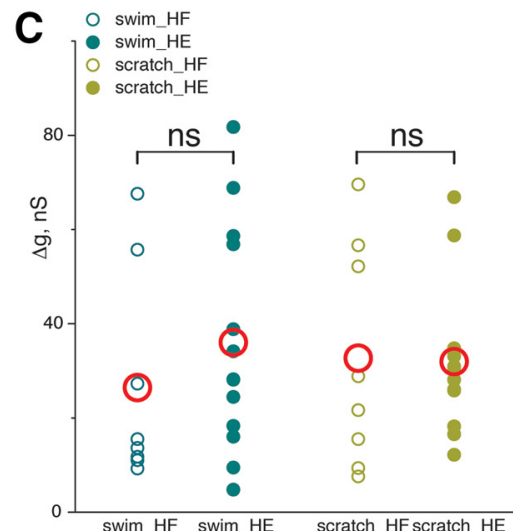

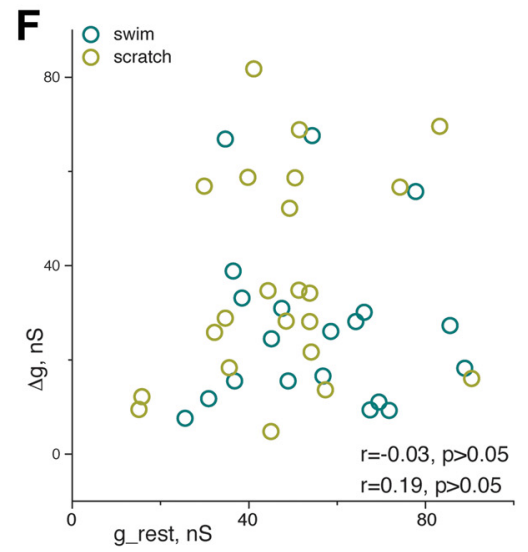

Figure 7. Conductance increase during swimming and scratching. All data points above diagonal in $A$ indicate that conductance increases in all motoneurons during swimming (blue circles) and scratching (yellow circles). B, Similar conductance increase during swimming and scratching (red circles represent mean). C, Similar conductance increase in HF and HE motoneurons during swimming and scratching (red circles represent mean). D, Similar conductance increase in spiking and nonspiking motoneurons during swimming and scratching (red circles represent mean). $\boldsymbol{E}$, Conductance increase during swimming correlates with conductance increase during scratching. $F$, No correlation between resting conductance and increased conductance during swimming and scratching. ns, Not significant $(p>0.05)$.

Significant increases in conductance during network activity have been demonstrated in cortical neurons (Dreifuss et al., 1969; Borg-Graham et al., 1998; Wehr and Zador, 2003). In contrast, insignificant or small changes in input conductance have been reported for motoneurons during locomotor activity (Shefchyk and Jordan, 1985; MacDonell et al., 2015). It has also been shown that conductance increases can be behavior-related (Perreault, 2002). Therefore, one may speculate that a conductance increase during scratching in turtle motoneurons (Robertson and Stein, 1988; Alaburda et al., 2005; Berg et al., 2008) could be specific for scratching rather than a general feature of motor network activity. In this study, we demonstrate that conductance of motoneurons increases during both scratching and swimming. The conductance increase during swimming and scratching observed in this study $(\sim 60 \%)$ is comparable with previous findings during scratching (Alaburda et al., 2005). Conductance increase was found not only in spiking but also nonspiking motoneurons. This could indicate that motoneurons with different excitability receive common input and that information coding in turtle spinal network is dense and distributed (Guzulaitis et al., 2014).

We found no relation between the resting conductance and the conductance increase during network activity. This suggests that the relative conductance increase during network activity is determined by other factors than the cell size. In addition, the relation between the resting conductance and the conductance increase during network activity may be quenched by the artifac- tual shunt induced by the recording electrode, particularly in small cells ( $\mathrm{Li}$ et al., 2004). We also found that the conductance increase for a given motoneuron was surprisingly similar during scratching and swimming. This could indicate that individual motoneurons are recruited by shared premotor network during different behaviors (Berkowitz et al., 2010). Alternatively, similar conductance increase during different behavior might signal a characteristic intrinsic conductance mechanism activated in motoneurons during network activity.

In conclusion, we demonstrated that turtle motoneurons are activated very similarly during two distinct behaviors (i.e., scratching and swimming). Even though these behaviors are initiated in very different manners and produce qualitatively different motor patterns, motoneurons are engaged very similarly by the premotor network in terms of synaptic intensity and postsynaptic firing pattern. We find that motoneurons receive intense synaptic input during swim and scratch episodes, as reflected in the conductance increase, $V_{\mathrm{m}}$ fluctuations, and irregular firing.

\section{References}

Alaburda A, Hounsgaard J (2003) Metabotropic modulation of motoneurons by scratch-like spinal network activity. J Neurosci 23:8625-8629. Medline

Alaburda A, Russo R, MacAulay N, Hounsgaard J (2005) Periodic highconductance states in spinal neurons during scratch-like network activity in adult turtles. J Neurosci 25:6316-6321. CrossRef Medline 
Azouz R, Gray CM (1999) Cellular mechanisms contributing to response variability of cortical neurons in vivo. J Neurosci 19:2209-2223. Medline

Berg RW, Alaburda A, Hounsgaard J (2007) Balanced inhibition and excitation drive spike activity in spinal half-centers. Science 315:390-393. CrossRef Medline

Berg RW, Ditlevsen S, Hounsgaard J (2008) Intense synaptic activity enhances temporal resolution in spinal motoneurons. PLoS One 3:e3218. CrossRef Medline

Berkowitz A, Roberts A, Soffe SR (2010) Roles for multifunctional and specialized spinal interneurons during motor pattern generation in tadpoles, zebrafish larvae, and turtles. Front Behav Neurosci 4:36. CrossRef Medline

Borg-Graham LJ, Monier C, Frégnac Y (1998) Visual input evokes transient and strong shunting inhibition in visual cortical neurons. Nature 393: 369-373. CrossRef Medline

Delgado-Lezama R, Hounsgaard J (1999) Adapting motoneurons for motor behavior. Prog Brain Res 123:57-63. CrossRef Medline

Destexhe A, Rudolph M, Paré D (2003) The high-conductance state of neocortical neurons in vivo. Nat Rev Neurosci 4:739-751. CrossRef Medline

Dreifuss JJ, Kelly JS, Krnjeviæ K (1969) Cortical inhibition and gammaaminobutyric acid. Exp Brain Res 9:137-154. CrossRef Medline

Faisal AA, Selen LP, Wolpert DM (2008) Noise in the nervous system. Nat Rev Neurosci 9:292-303. CrossRef Medline

Frigon A, Gossard JP (2010) Evidence for specialized rhythm-generating mechanisms in the adult mammalian spinal cord. J Neurosci 30:70617071. CrossRef Medline

Gandevia SC, Macefield G, Burke D, McKenzie DK (1990) Voluntary activation of human motor axons in the absence of muscle afferent feedback: the control of the deafferented hand. Brain 113:1563-1581. CrossRef Medline

Grillner S (2003) The motor infrastructure: from ion channels to neuronal networks. Nat Rev Neurosci 4:573-586. CrossRef Medline

Grillner S, Parker D, el Manira A (1998) Vertebrate locomotion: a lamprey perspective. Ann N Y Acad Sci 860:1-18. CrossRef Medline

Guzulaitis R, Alaburda A, Hounsgaard J (2013) Increased activity of premotor network does not change the excitability of motoneurons during protracted scratch initiation. J Physiol 591:1851-1858. CrossRef Medline

Guzulaitis R, Alaburda A, Hounsgaard J (2014) Dense distributed processing in a hindlimb scratch motor network. J Neurosci 34:10756-10764. CrossRef Medline

Holt GR, Softky WR, Koch C, Douglas RJ (1996) Comparison of discharge variability in vitro and in vivo in cat visual cortex neurons. J Neurophysiol 75:1806-1814. Medline

Hounsgaard J, Hultborn H, Jespersen B, Kiehn O (1988) Bistability of alpha-motoneurones in the decerebrate cat and in the acute spinal cat after intravenous 5-hydroxytryptophan. J Physiol 405:345-367. CrossRef Medline

Juranek J, Currie SN (2000) Electrically evoked fictive swimming in the lowspinal immobilized turtle. J Neurophysiol 83:146-155. Medline

Kiehn O, Kjaerulff O, Tresch MC, Harris-Warrick RM (2000) Contributions of intrinsic motor neuron properties to the production of rhythmic motor output in the mammalian spinal cord. Brain Res Bull 53:649-659. CrossRef Medline

Leitch M, Macefield VG (2014) Comparison of the contractile responses to irregular and regular trains of stimuli during microstimulation of single human motor axons. J Neurophysiol 111:1499-1506. CrossRef Medline

Lennard PR, Stein PS (1977) Swimming movements elicited by electrical stimulation of turtle spinal cord: I. Low-spinal and intact preparations. J Neurophysiol 40:768-778. Medline

Li WC, Soffe SR, Roberts A (2004) A direct comparison of whole cell patch and sharp electrodes by simultaneous recording from single spinal neurons in frog tadpoles. J Neurophysiol 92:380-386. CrossRef Medline

MacDonell CW, Power KE, Chopek JW, Gardiner KR, Gardiner PF (2015) Extensor motoneurone properties are altered immediately before and during fictive locomotion in the adult decerebrate rat. J Physiol 593:2327-2342. CrossRef Medline

Macefield VG, Gandevia SC, Bigland-Ritchie B, Gorman RB, Burke D (1993) The firing rates of human motoneurones voluntarily activated in the absence of muscle afferent feedback. J Physiol 471:429-443. CrossRef Medline

Marder E, Bucher D (2007) Understanding circuit dynamics using the stomatogastric nervous system of lobsters and crabs. Annu Rev Physiol 69: 291-316. CrossRef Medline

Marder E, Calabrese RL (1996) Principles of rhythmic motor pattern generation. Physiol Rev 76:687-717. Medline

Monier C, Fournier J, Frégnac Y (2008) In vitro and in vivo measures of evoked excitatory and inhibitory conductance dynamics in sensory cortices. J Neurosci Methods 169:323-365. CrossRef Medline

Mortin LI, Keifer J, Stein PS (1985) Three forms of the scratch reflex in the spinal turtle: movement analyses. J Neurophysiol 53:1501-1516. Medline

Noda H, Adey WR (1970) Firing variability in cat association cortex during sleep and wakefulness. Brain Res 18:513-526. CrossRef Medline

Paré D, Shink E, Gaudreau H, Destexhe A, Lang EJ (1998) Impact of spontaneous synaptic activity on the resting properties of cat neocortical pyramidal neurons in vivo. J Neurophysiol 79:1450-1460. Medline

Perreault MC (2002) Motoneurons have different membrane resistance during fictive scratching and weight support. J Neurosci 22:8259-8265. Medline

Petersen PC, Vestergaard M, Jensen KH, Berg RW (2014) Premotor spinal network with balanced excitation and inhibition during motor patterns has high resilience to structural division. J Neurosci 34:2774-2784. CrossRef Medline

Robertson GA, Stein PS (1988) Synaptic control of hindlimb motoneurones during three forms of the fictive scratch reflex in the turtle. J Physiol 404:101-128. CrossRef Medline

Ruigrok TJ, Crowe A (1984) The organization of motoneurons in the turtle lumbar spinal cord. J Comp Neurol 228:24-37. CrossRef Medline

Salinas E, Sejnowski TJ (2000) Impact of correlated synaptic input on output firing rate and variability in simple neuronal models. J Neurosci 20: 6193-6209. Medline

Shadlen MN, Newsome WT (1998) The variable discharge of cortical neurons: implications for connectivity, computation, and information coding. J Neurosci 18:3870-3896. Medline

Shefchyk SJ, Jordan LM (1985) Motoneuron input-resistance changes during fictive locomotion produced by stimulation of the mesencephalic locomotor region. J Neurophysiol 54:1101-1108. Medline

Softky WR, Koch C (1993) The highly irregular firing of cortical cells is inconsistent with temporal integration of random EPSPs. J Neurosci 13: 334-350. Medline

Stein PS (2005) Neuronal control of turtle hindlimb motor rhythms. J Comp Physiol A Neuroethol Sens Neural Behav Physiol 191:213-229. CrossRef Medline

Vaillancourt DE, Larsson L, Newell KM (2003) Effects of aging on force variability, single motor unit discharge patterns, and the structure of 10 , 20, and $40 \mathrm{~Hz}$ EMG activity. Neurobiol Aging 24:25-35. CrossRef Medline

Wehr M, Zador AM (2003) Balanced inhibition underlies tuning and sharpens spike timing in auditory cortex. Nature 426:442-446. CrossRef Medline 